\title{
Analysis of anti-radical activity of some food suitable algae of the sea of Okhotsk
}

\author{
Viktor Gorbachev ${ }^{1,2}$, Mariya Klokonos ${ }^{1}$, Olga Orlovtseva $^{1}$, Svetlana Tefikova ${ }^{1}$, and \\ Igor Nikitin ${ }^{1 *}$ \\ ${ }^{1}$ K.G. Razumovsky Moscow State University of technologies and management \\ (The First Cossack University), St. Zemlyanoy Val, 73, Moscow, Russian Federation \\ ${ }^{2}$ FGBNU Magadan Research Institute of Agriculture, St. Proletarskaya, 17, Magadan, Russian \\ Federation
}

\begin{abstract}
The antiradical activity (ARA) of 11 species of macrophyte algae in the northern part of the Sea of Okhotsk was analyzed for the first time. The highest ARA indicators were established for cystozera, ulva and fucus and exceeded the traditionally eaten kelp by 1.2-2.2 times. Oil infusions of kelp showed an increase in ARA scores by $13 \%$ compared to its alcoholic extracts. Average ARA values decreased in the following sequence: brown (with scatter of data), green, red algae. Despite the low ARA values, in red algae $(\approx 33 \%$ of the maximum value), the effect of an increase in the viscosity of hot solutions was found when using algae of the genus Chondrus, which, in our opinion, was explained by the presence of caraginans. Assessment of the fall in ARA during storage of dried products from whole algae revealed a decrease in the value of this parameter $\approx 2.5 \%$ per month.
\end{abstract}

\section{Introduction}

The human body, as well as animals, constantly generates free radicals, which, on the one hand, are capable of intensifying the course of pathological conditions, and on the other hand, are a consequence of their course [1]. The use of substances capable of trapping radicals in the body is a traditional part of therapeutic measures both in the treatment of "free radical" pathologies, and in the correction of nutrition of the population living in remote areas with a poor food base, in ecologically unfavorable regions, when working in hazardous industries and in the presence of the same type of food addictions in humans $[2,4]$.

In light of the above, it becomes relevant both to search for new sources of food raw materials (non-traditional food raw materials) with high antiradical activity (hereinafter ARA), and to compare them with already used common food products in one comparable category [5].

Most of the population in northeastern Russia (Magadan Region, Chukotka, and Kamchatka Territory) lives in the coastal area [3], which determines the demand for aquatic biological resources, including algae. Additional factors are the proximity of the countries

\footnotetext{
* Corresponding author: nikitinia@mgutm.ru
} 
of the Asia-Pacific region (Japan, Korea, China, etc.), and the growing popularity of Japanese cuisine, where sea plants are traditional components of dishes.

Previously, the literature provided data on the high ARA and on the nutritional value of algae from the southern waters of the Far Eastern and Asian seas [6]. In particular, for example, works have been published on the superior concentrations of $\beta$-carotene (provitamin A) in green algae of lettuce (Ulva lactuca) in relation to carrots by more than 22 times [7]. The results of the analysis of the hepatoprotective properties of extracts from algae and the possibility of their use for the prevention of stress-induced metabolic disorders were also published [8-10] and so on.

Despite the revealed advantages and high nutritional values in algae [6], in Russia the possibility of their use for the food industry is still not sufficiently studied. Except for the production of agar and carrageenans (some of which are exported), the main and most accessible product for consumers from this group is seaweed (Sacharina sp).

One of the methods for the general assessment of the suitability of food raw materials and its value is the determination of the ARA [11]. On the one hand, a direct dependence of high APA values on the presence of high concentrations of polysaccharides, carotenoids, ascorbic acid, tocopherols and structurally similar polyphenol compounds of flavonoids has been shown [12]. On the other hand, high APA values indicate the possibility of an extended shelf life without a significant drop in consumer properties, both for raw materials and for processed products due to slowed down oxidation processes of food components [7].

One of the oldest and most well-known approaches is the assessment of ARA using the free radical N-diphenyl-picryl-gindrazyl (DPPH). The reduced and colored forms of this radical differ in transmission capacity in the blue-violet region, which makes it possible to estimate the amount of the interacting substance by the optical density value. A similar approach has been used with success to evaluate ARA [13, 14].

Based on the foregoing, the purpose of our study is to evaluate and compare the ARA of potentially suitable for food processing algae (macrophytes) in the northern part of the Sea of Okhotsk, based on the optical density obtained during the colored DPPH reaction.

\section{Material and methods}

The material was thalli of algae collected in the late autumn period in the intertidal zone in the waters near Magadan. The analysis used primarily mass plant species that form a large thallus [14]. The collected samples were washed, removed from impurities and dried in two temperature modes: for extraction at $60{ }^{\circ} \mathrm{C}$, and for determination of dry weight at $110{ }^{\circ} \mathrm{C}$ for 24 hours. At the end of drying, the samples were roughly ground in a mortar, weighed out, and poured into pharmaceutical bottles with a dense, ground-in soft rubber stopper. The solvent used was $70^{\circ}$ ethyl alcohol with a dry matter to solvent ratio of 1:20. The samples were infused at $-20^{\circ} \mathrm{C}$ for a week with occasional stirring. To eliminate the selfoxidation of antiradical fractions, the empty volume in the bottles was purged with a fivefold volume of carbon dioxide, which was fed into the bottle through a long medical probe, and the excess air was removed from the bottle through a second shorter probe. The removed air was tested for its ability to oxidize by means of a qualitative chemical reaction - the introduction of a burning flame into its stream, which was extinguished in the event of a complete replacement of the air volume with carbon dioxide inside the bottle. Already developed products from seaweed were used as reference groups - sheets for sushi (nori) and seaweed for soups (miyok) produced in South Korea, Busan. We also compared the kinetics of decolorization of solutions using standard substances with lipophilic and hydrophilic properties (see next chapter). 
The measurements were carried out on a KFK concentration photocolorimeter, taking into account the transmission width of the wave from 515 to $565 \mathrm{~nm}$, which falls on the DPPG absorption maximum at $517 \mathrm{~nm}$. To measure the optical density of the products of the bleaching reaction, we used a solution of DPPH in $96^{\circ}$ ethyl alcohol, with a concentration of $1.27 * 10-4 \mathrm{~mol} /$ liter, which was stored in a dark place at $-20^{\circ} \mathrm{C}$.

The analysis was carried out taking into account the negative (without discoloration with the addition of $0.5 \mathrm{ml}$ of pure alcohol) and positive control (with the addition of $0.5 \mathrm{ml}$ of a 0.01 molar alcohol solution of ascorbic acid). The volume of the reaction mixture was $2.5 \mathrm{ml}, 2 \mathrm{ml}$ of which were for the calibrated DPPH solution, and $0.5 \mathrm{ml}$ for the analyzed extract, as we have already described [5]. The exposure time of the solution was 30 minutes in a dark place. All samples were analyzed 5 times, while the values were rechecked 3 times, taking into account the instrument error of $0.3 \%$. In accordance with the recommendations given earlier in the literature [13], as 1 equivalent was taken $\mu$ mol of ascorbic acid.

For the DPPH solution, calibration was carried out using a series of dilutions of ascorbic acid from $1 * 10-6$ to $1 * 10-8 \mathrm{~mol} / 1$, based on the data obtained, a graph of $50 \%$ decoloration of the solution was plotted, which was $4.24 * 10-7$ mol per reaction ... Taking into account the range of values, by analogy with earlier studies, we calculated the dependence of the change in optical density, which was $\approx 0.0062$ per $\mathrm{cm} / \mathrm{eq} * 10-2$ (per centi-equivalent further seq or eq $* 10-2)$. Based on the above data and the difference in optical density of the analyzed samples, the active substance with antiradical activity was recalculated per gram of dry weight of algae.

Additionally, we also tested the assumptions about the possibility of using oil extracts of seaweed using the example of kelp. For this, an infusion was made on refined, nondeodorized vegetable oil instead of alcohol. The volumes of the weighed samples and the ratio of the solvent and the extraction time were kept in the same proportion as for the aqueous-alcoholic extracts. Before optical measurement, the antiradical fractions were overextracted from the oil with $96 \%$ ethyl alcohol, which was already used in the reaction. A sample of the purest oil was used as a negative control in the analysis of fat extracts. The difference in activity (delta) was determined by the ratio of APA values for pure oil and extracts based on it. The statistical calculation was carried out in Excel 2010.

\section{Results and discussion}

DPPH is known to be able to deprotonate various functional groups in molecules [12]. It interacts not only with hydrophilic, but also lipophilic components of food systems. A similar mechanism manifests itself in relation to free radicals generated in the human body during biochemical reactions.

To assess the rate of kinetics, standard, pure substances used for the food and pharmaceutical industries were used: tocopherol (21st Century), lecithin (Now foods), a mixture of polyunsaturated fatty acids (docosahexaenoic and eicosopentaenoic in a 2: 1 ratio) produced from algae (Source naturals, Vegan), ascorbic acid (NutriBiotic) and extracts from fireweed of narrow-leaved production of local residents of the Magadan region, as previously published [15].

The main trends in discoloration of reaction mixtures are shown in Figure 1. On the left side of the graph, as you can see, the trend corresponding to extracts from algae forms a broken curve, changing the slope from about $45^{\circ}$ to $83^{\circ}$ (orange trend line). It is easy to see that the slopes of the bleaching trends for hydrophilic and lipophilic groups of substances differ in their curvature, as substances from the first group react sharply with free radicals, including due to their higher ability to dissociate. 


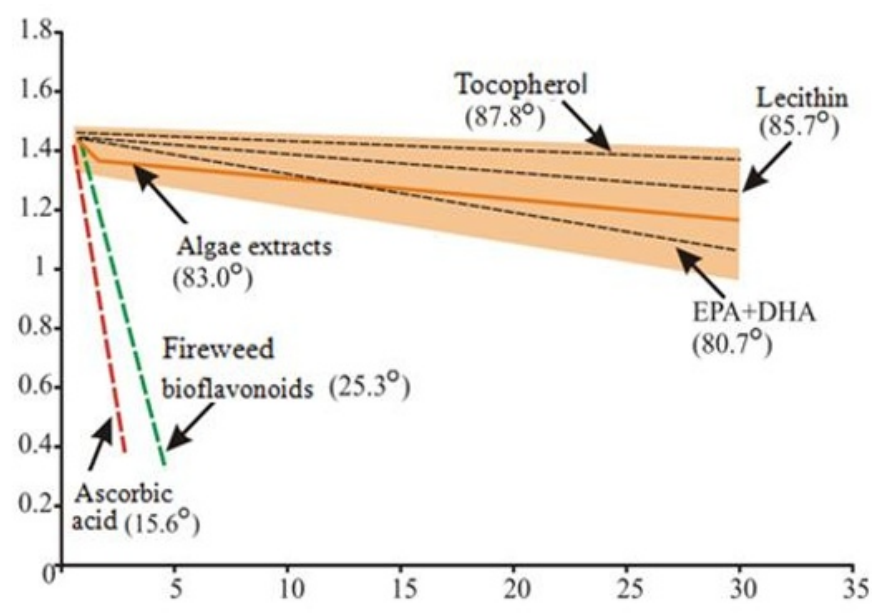

Fig. 1. Trends of discoloration of a standardized DPPH solution with extracts from algae, lipophilic and hydrophilic substances. Note: in parentheses next to the captions, the slope angles of the trends, calculated through their cotangents $(\operatorname{ctg} \alpha)$; the filled trapezoidal area on the graph is the scatter of values for the trends in the reactivity of extracts from algae. The ordinate is the optical density, the abscissa is the exposure time in minutes.

The trends for algal extracts show similarities in different parts of the graph for both groups, indicating that they are mixed. As the rapidly reacting part of antiradical substances is depleted, the trend on the graph changes its curvature (Fig. 1). Moreover, it crosses the trend for polyunsaturated fatty acids, which indicates their presence in the original extract, and depletion of this type of substance by the end of the reaction. Thus, we can reasonably assume that substances with antiradical activity react mainly in the following order: hydrophilic group (vitamin $\mathrm{C}$, bioflavonoids), then reactive lipophilic fractions (eicosopentaenoic and docosahexaenoic acids), and last of all membrane lipids (lecithins) and tocopherols.

It should be noted that we found a direct correlation between the optical density (solution extinction) of undiluted algal extracts and the final rate of DPPH discoloration. Figure 2 shows the probability density distribution of the correlation values between these parameters.

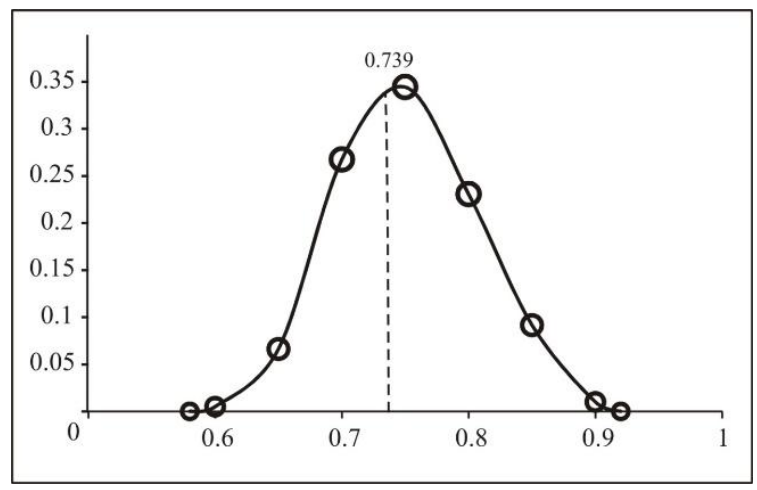

Fig. 2. Distribution of the probability density of correlations between the value of discoloration of the calibrated DPPH solutions and the optical density of the initial extracts from algae at 1000 bootstrap iterations of the experimental data. 


\section{EEESTS-2021}

On average, the probability density for 1000 simulations by the resampling method (bootstrap / Bootstrap) was about 74\%, which makes it possible to propose measurement of the optical density of algae extracts without the use of other reagents as an express test for approximate APA estimation.

The APA values themselves are presented in the table 1. Average values are shown as a bar chart in Figure 3, in order of increasing.

Table 1. Antiradical activity of algae extracts and products of their processing in equivalents per gram of dry weight in a $95 \%$ confidence interval.

\begin{tabular}{|c|c|c|}
\hline № & Algae name / genus name in Latin & APA, eq/g CI, 95\% \\
\hline \multicolumn{3}{|c|}{ Brown algae } \\
\hline 1. & $\begin{array}{l}\text { Alaria Okhotsk } \\
\text { Alaria }\end{array}$ & $96,7-101,7$ \\
\hline 2. & $\begin{array}{l}\text { Kelp Ribbon-like } \\
\text { Sacharina }\end{array}$ & $207,8-214,5$ \\
\hline 3. & $\begin{array}{l}\text { Kelp adressorizoid } \\
\text { Sacharina }\end{array}$ & $101,1-130,0$ \\
\hline 4. & $\begin{array}{l}\text { Kelp oblique } \\
\text { Sacharina }\end{array}$ & $87,9-89,9$ \\
\hline 5. & $\begin{array}{l}\text { Pseudolessonia kelp } \\
\text { Pseudolessonia }\end{array}$ & $83,1-86,1$ \\
\hline 6. & $\begin{array}{l}\text { Cystoseira thick-legged } \\
\text { Cystoseira }\end{array}$ & $433,3-471,9$ \\
\hline 7. & $\begin{array}{l}\text { Fucus vanishing } \\
\text { Fucus }\end{array}$ & $246,4-256,8$ \\
\hline \multicolumn{3}{|c|}{ Red algae } \\
\hline 8. & $\begin{array}{l}\text { Neohypophyllum Middendorf } \\
\text { Neohypophyllum }\end{array}$ & $134,0-143,8$ \\
\hline 9. & $\begin{array}{l}\text { Porphyra changeable } \\
\text { Porphyra }\end{array}$ & $129,8-137,5$ \\
\hline 10. & $\begin{array}{l}\text { Chondrus flat } \\
\text { Chondrus }\end{array}$ & $138,6-156,1$ \\
\hline \multicolumn{3}{|c|}{ Green algae } \\
\hline 11. & $\begin{array}{l}\text { Ulva brilliant } \\
\text { Ulva }\end{array}$ & $326,0-374,7$ \\
\hline \multicolumn{3}{|c|}{$\begin{array}{l}\text { Refined products for comparison } \\
\end{array}$} \\
\hline \multicolumn{3}{|c|}{ Brown algae } \\
\hline 12. & $\begin{array}{l}\text { Dried seaweed «miek / wakame» for soups } \\
\text { made in Korea, Busan } \\
\text { Sin Young Food Co. } \\
\text { Undaria sp }\end{array}$ & $19,7-20,7^{*}$ \\
\hline \multicolumn{3}{|c|}{ Red algae } \\
\hline 13. & $\begin{array}{l}\text { Seaweed «nori / gimbab gim» in sheets for } \\
\text { making sushi made in Korea, Busan } \\
\text { Sin Young Food Co. } \\
\text { Porphyra sp }\end{array}$ & $16,0-17,7 *$ \\
\hline
\end{tabular}

As you can see from the table, and likewise from the histogram presented below, the algae processing products we took for analysis had extremely low ARA values. All the samples we collected exceeded the nori algae in ARA by an average of 11.22 times. We also note that three types of algae (cystozeira, fucus and ulva) surpass in antiradical potential the seaweed (kelp) traditionally used in food, which makes it promising to develop new recipes for salads and other dishes with or on their basis (Figure 3). 


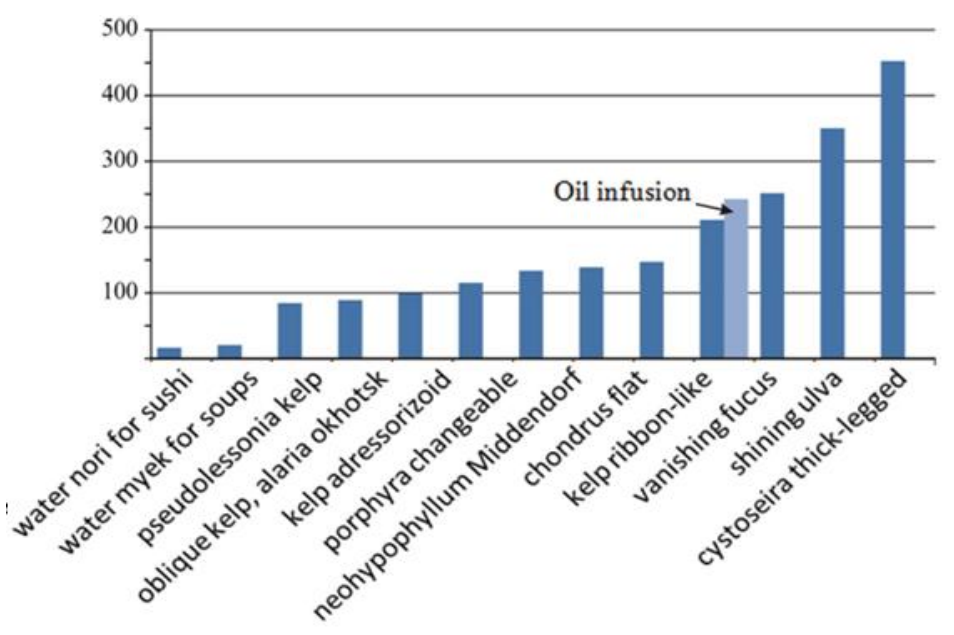

Fig. 3. ARA histogram of the studied samples in order of increasing. Note: the ordinate is the number of equivalents / $g$ dry weight.

We point out that the production dates for each of the products varied greatly. So nori algae was developed in August 2020, and miyok algae in May 2017. Even though their shelf life is over, their residual activity is higher than that of land algae. We associate this not only with the initial antiradical potential of the algae themselves, but also with the area of contact of these products with atmospheric oxygen, because to obtain nori, plant raw materials are mechanically processed and pressed into sheets, which greatly increases the oxidation of residual fractions with ARA. Miyok algae are stored as a whole, which reduces the rate of their interaction with atmospheric oxygen even during long-term storage.

Taking into account all of the above and the fact that both the storage time interval and the confidence interval of ARA are known for all studied species of algae, it is possible to estimate the fall of ARA to date. The shelf life of these products was approximately 42 months, with a 95\% confidence interval for APA in the range of 97 to 238 equiv / gram of dry weight.

It follows that the potential decreases by about $2.4 \%$ per month for dry, non-fragmented algae (from 2.3 to $5.7 \mathrm{eq} / \mathrm{month}$ ), and for products in the form of dried nori sheets, it is 10 times higher than about 25\%. Korean manufacturers indicate that the shelf life of this product is 12 months, but we believe that the shelf life should be reduced to half a year.

In our opinion, it is quite surprising that the values of ARA within the genus of kelp accept such a large scatter. We do not exclude, however, the fact that the final values were influenced by the time of collecting the material. It is possible that the samples collected in the spring-summer period will show a different dynamics.

In general, despite the scatter of values within the group of brown algae, it has a high potential, including for use in processing, and equally for food in the form of mixtures of thalli of these plants. In our opinion, the addition of ground dried or pasty mixtures of both brown and green algae in the production of bakery and confectionery products, including in the form of preserves, is also very promising. As can be seen from the ARA table, the activities of the algae groups are arranged in the following order: brown algae, green, red.

We also note that the assessment of ARA oil infusion of kelp in relation to the activity of the sample of the vegetable oil itself showed an increase in this parameter in the range of $12.9-13.9 \%$. It can also be seen on the histogram that the kelp oil infusion shows a higher APA value compared to alcoholic extracts. We attribute this to the synergistic effect of both the components of the «seaweed» itself and the residual concentrations of tocopherols from the oil. A similar effect makes it possible to use vegetable oils for the extraction of 
biologically active substances from algae, including for increasing the ARA of food products, for example, in the production of sauces, fillings, flour products and preserves with an increased shelf life.

Despite the lower APA values in red algae, we found an increase in the viscosity of hot aqueous infusions and decoctions of Chondrus flata algae, as we believe, due to the high content of carrageenan. In view of the fact that other types of chondrus from the seas of Europe are used to extract several types of sulfated polysaccharides of carrageenans, which are often used in dairy, meat and confectionery industries, we assumed that this direction will be promising in the future for algae from the seas of the Far East.

\section{Conclusions}

Summing up, it is important to note that, in our opinion, both the food and resource potentials of the marine plants of the Far East have not been fully studied. Expanding the range of algae used in food can successfully diversify both the insufficient mineral and vitamin base of the inhabitants of the Far North and remote territories of Russia, and adjust to a large extent the small anti-radical potential of refined food. The complex dynamics of discoloration of DPPH solutions gives grounds to consider the presence in algae and products of their processing of several groups of compounds with different reactivity, which, in our opinion, will increase the shelf life and stability of food systems when these components are introduced into food formulations.

\section{References}

1. J. Pincemail, Analysis of Free Radicals in Biological Systems. Birkhäuser Basel. 8398 (1995)

2. I.E. Ionova, Peculiarities of the nature of nutrition and health of the indigenous (smallnumbered) and newcomer population of the Far North, (Thesis Ph.D. of med. sciences. Moscow, 2004)

3. A.P. Bulban, Comparative ecological and physiological characteristics of the microelement status of the population of the coastal and continental territories of the Magadan region, (Thesis Ph.D.of biol. sciences. Magadan, 2005)

4. V.I. Karpov, N.M. Portnov, I.A. Nikitin, Y.I. Sidorenko, I.V. Zavalishin, S.M. Petrov, N.M. Podgornova, M.Y. Sidorenko, S.V. Shterman, International Journal of Advanced Computer Science and Applications. 10 (11), 317-322 (2019)

5. A.G. Lapinsky, V.V. Gorbachev Chemical and pharmaceutical journal. 40, 6, 27-29 (2006)

6. N.G. Klochkova, V.A. Berezovskaya, Algae of the Kamchatka shelf. Distribution, biology, chemical composition, (Vladivostok; Petropavlovsk-Kamchatsky: Dalnauka, 1997)

7. H.H. El-Baky, F.K. Baz, S.E. Baroty, American-Eurasian J. Agric. \& Environ. Sci. 3 (3), 434-444 (2008)

8. T.A. Kuznetsova, I.G. Agafonova, T.S. Krokhmal, T.N. Zvyagintseva, N.V. Filonova, Pacific Medical Journal. 4 (42), 32-35 (2010)

9. N.F. Kushnerova, Health. Medical ecology. The science. 4 (71), 101-103 (2017)

10. S.E. Fomenko, Health. Medical ecology. Science. 4 (71), 126-128 (2017)

11. S. Fomenko, N. Kushnerova, V. Sprygin, E. Drugova, L. Lesnikova, V. Merzlyakov, T. Momot, Plant Physiology. 66, 452-460 (2019) 
12. C.A. Rice-Evans, A.T. Diplock, M.C.R. Symons, Laboratory techniques in biochemistry and molecular biology. Techniques in Free Radical Research. Elsevier, 309 (1991)

13. J. Glavind, Acta chemica scandinavica. 17, 1635-1640 (1963)

14. T.A. Klochkova, M.N. Bely, N.G. Klochkova, Algae-macrophytes of the northern part of the Sea of Okhotsk and their importance as a spawning substrate of herring, (Magadan: New printing industry, 2013)

15. T.A. Gorbacheva, New Kolyma. Lead. 53 (657), 14 (2018) 